Pacific Journal of Mathematics

A SPECTRAL DUALITY THEOREM FOR CLOSED 


\section{A SPECTRAL DUALITY THEOREM FOR CLOSED OPERATORS}

\section{ERdelyi AND WANG SHENGWANG}

The spectral duality theorem asserts that a densely defined closed operator $T$ induces a spectral decomposition of the underlying Banach space $X$ iff the conjugate $T^{*}$ induces the same type of spectral decomposition of the dual space $X^{*}$. This theorem is known for bounded linear operators in terms of residual $(S)$-decomposability. In this paper we extend the spectral duality theorem to unbounded operators, under a general type of spectral decomposition. Our approach to the spectral duality leads through the successive conjugates $T^{*}, T^{* *}$ and $T^{* * *}$ of $T$, under their domain-density assumptions.

1. Elements of local spectral theory for a closed operator. $X$ is an abstract Banach space over the complex field C. If $S$ is a set, we write $\bar{S}$ for the closure, Int $S$ for the interior, $S^{c}$ for the complement, $\partial S$ for the boundary, and $\operatorname{cov} S$ for the collection of all finite open covers of $S$. If $S$ is a subset of $\mathbf{C}$, then the above mentioned topological constructs are referred to the topology of $\mathbf{C}$. Without loss of generality, we assume that for $S \subset \mathbf{C}$, each $\left\{G_{l}\right\}_{l=0}^{n} \in \operatorname{cov} S$ has, at most, one unbounded set $G_{0}$. An open $G \subset \mathbf{C}$ is said to be a neighborhood of $\infty$, in symbols $G \in V_{\infty}$, if for $r>0$ sufficiently large, $\{\lambda \in \mathbf{C}:|\lambda|>r\} \subset G$. We write $S^{\perp}$ for the annihilator of $S \subset X$ in $X^{*}$ (as well as that of $S \subset X^{* *}$ in $X^{* * *}$ ) and ${ }^{\perp} S$ for the preannihilator of $S \subset X^{*}$ in $X$ (or that of $S \subset X^{* * *}$ in $X^{* *}$ ). $B(X)$ denotes the Banach algebra of all bounded linear operators which map $X$ into $X$. $I$ stands for the identity operator.

For a linear operator $T: D_{T}(\subset X) \rightarrow X$, we use the following notations: spectrum $\sigma(T)$, resolvent set $\rho(T)$, and resolvent operator $R(\cdot ; T)$.

If $T$ has the single valued extension property (SVEP) then, for $x \in X$, $\sigma_{T}(x)$ is the local spectrum, $\rho_{T}(x)$ is the local resolvent set and $x(\cdot)$ is the local resolvent function. For $S \subset \mathbf{C}$, an extensive use will be made of the spectral manifold $X(T, S)=\left\{x \in X: \sigma_{T}(x) \subset S\right\}$.

Inv $T$ represents the lattice of all invariant subspaces under $T$. For $Y \in \operatorname{Inv} T, T \mid Y$ is the restriction of $T$ to $Y$ and $T / Y$ denotes the coinduced operator on the quotient space $X / Y$ with domain $D_{T / Y}=\{\hat{x} \in$ $\left.X / Y: \hat{x} \cap D_{T} \neq \varnothing\right\}$.

If not mentioned otherwise, throughout this paper $T$ is a densely defined unbounded closed operator with domain and range in $X$. 
Given $T$, the following domain-density conditions will guarantee the existence of the successive conjugates:

(*) $T^{*}$ is densely defined;

(**) $T^{*}$ and $T^{* *}$ are densely defined;

(***) $T^{*}, T^{* *}$ and $T^{* * *}$ are densely defined.

With $J$ and $K$, the natural embeddings of $X$ into $X^{* *}$ and of $X^{*}$ into $X^{* * *}$, respectively, we shall explore the direct sum decomposition

$$
X^{* * *}=K X^{*} \oplus(J X)^{\perp} \text {. }
$$

For completeness, we give a short proof of (1.1), (e.g. [10]). For every $x^{* * *} \in X^{* * *}$, one defines $x^{*} \in X^{*}$ by

$$
\left\langle x, x^{*}\right\rangle=\left\langle J x, x^{* * *}\right\rangle, \quad x \in X .
$$

Then, $\left\langle J x, K x^{*}\right\rangle=\left\langle x^{*}, J x\right\rangle=\left\langle x, x^{*}\right\rangle=\left\langle J x, x^{* * *}\right\rangle$ and hence $y^{* * *}=$ $x^{* * *}-K x^{*} \in(J X)^{\perp}$. This, together with $K X^{*} \cap(J X)^{\perp}=\{0\}$, establishes (1.1).

The spectral theoretic results will be expressed in terms of operators with the spectral decomposition property, decomposable operators and $\{\infty\}$-decomposable operators.

1.1. Definition. $T$ is said to have the spectral decomposition property (SDP) if, for any $\left\{G_{\imath}\right\}_{l=0}^{n} \in \operatorname{cov} \sigma(T)$ with $G_{0} \in V_{\infty}$, there is a system $\left\{Y_{l}\right\}_{l=0}^{n} \subset$ Inv $T$ satisfying the following conditions:

(I) $Y_{i} \subset D_{T}$ if $G_{l}$ is relatively compact in $\mathbf{C}(1 \leq i \leq n)$;

(II) $X=\sum_{l=1}^{n} Y_{i}$ and $\sigma\left(T \mid Y_{l}\right) \subset G_{i}, 0 \leq i \leq n$.

If we restrict $n$ to $n=1$ then $T$ is said to have the 1-SDP.

The concept of spectral maximal space [3] has two distinct extensions to the case of unbounded operators.

1.2. Definition. $Y \in \operatorname{Inv} T$ is called a spectral maximal space (SMS) of $T$ if, for any $Z \in \operatorname{Inv} T$, the inclusion $\sigma(T \mid Z) \subset \sigma(T \mid Y)$ implies $Z \subset Y$.

1.3. Definition. $Y \in \operatorname{Inv} T$ is said to be a $T$-bounded spectral maximal space ( $T$-bounded SMS) if

(i) $Y \subset D_{T}$;

(ii) for every $Z \in \operatorname{Inv} T, Z \subset D_{T}$ and $\sigma(T \mid Z) \subset \sigma(T \mid Y)$ imply $Z \subset Y$.

This concept appears in [8] under the name of maximal invariant space. Clearly, every SMS of $T$ is a $T$-bounded SMS. Conversely, however, not every $T$-bounded SMS is a SMS of $T$. In fact, if $Y$ is a $T$-bounded 
SMS and $Z \in \operatorname{Inv} T$ is not contained in $D_{T}$, then $\sigma(T \mid Z) \subset \sigma(T \mid Y)$ need not imply $Z \subset Y$. In the bounded case, the two concepts coincide.

The following properties of the spectral manifold $X(T, \cdot)$ for closed $T$ are analogous, in statement and proof, to the one for a bounded operator $[3,5]$.

1.4. Proposition. Let $T$ have the SVEP. If, for closed $F \subset \mathbf{C}, X(T, F)$ is closed then $X(T, F)$ is a SMS of $T$ and

$$
\sigma[T \mid X(T, F)] \subset F \cap \sigma(T) .
$$

Moreover, if $T$ has the 1-SDP then, for every closed $F \subset \mathbf{C}, X(T, F)$ is closed.

1.5. Proposition. Given $T$, let $Y \in \operatorname{Inv} T$ be such that $\sigma(T \mid Y)$ is compact in $\mathbf{C}$. There exist $\Upsilon, W \in \operatorname{Inv} T$ with the following properties:

(I) $Y=\Upsilon \oplus W, \sigma(T \mid \Upsilon)=\sigma(T \mid Y), \sigma(T \mid W)=\varnothing$;

(II) $\Upsilon \subset D_{T}$.

Proof. $\sigma_{1}=\sigma(T \mid Y)$ and $\sigma_{2}=\varnothing$ can be regarded as disjoint spectral sets of $\sigma(T \mid Y)$. Thus, the functional calculus produces (I). For a bounded Cauchy domain $\Delta \supset \sigma(T \mid Y), \Upsilon$ and $W$ can be expressed in terms of the spectral projection

$$
Q=\frac{1}{2 \pi i} \int_{\partial \Delta} R(\lambda ; T \mid Y) d \lambda
$$

(independent of the choice of $\Delta$ ) as follows: $\Upsilon=Q Y, W=\left(I_{Y}-Q\right) Y$, where $I_{Y}$ is the identity in $Y$. Since $T$ is closed, it follows easily that $\Upsilon$, $W \in \operatorname{Inv} T$ and $\Upsilon \subset D_{T}$.

1.6. TheOREM. Given $T$, let $Y$ be a SMS of $T$ with $\sigma(T \mid Y)$ compact in C. Then $\Upsilon$, as defined by Proposition 1.5, is a T-bounded SMS.

Proof. Let $Z \in \operatorname{Inv} T$ be such that $Z \subset D_{T}$ and

$$
\sigma(T \mid Z) \subset \sigma(T \mid \Upsilon)
$$

By Proposition 1.5, (1.4) implies $\sigma(T \mid Z) \subset \sigma(T \mid Y)$ and since $Y$ is a SMS of $T$, we have $Z \subset Y$. Then, for $x \in Z, \lambda \in \rho(T \mid Y)$, relation

$$
R(\lambda ; T \mid Z) x=R(\lambda ; T \mid Y) x
$$


implies

$$
Q x=\frac{1}{2 \pi i} \int_{\partial \Delta} R(\lambda ; T \mid Y) x d \lambda=\frac{1}{2 \pi i} \int_{\partial \Delta} R(\lambda ; T \mid Z) x d \lambda=x,
$$

where $\Delta \supset \sigma(T \mid Y)$ is a bounded Cauchy domain and $Q$ is the projection (1.3). Thus, we have $x=Q x \in \Upsilon$ and hence $Z \subset \Upsilon$.

The next theorem (partly adopted from [11]) gives some necessary and sufficient conditions for a $T$-bounded SMS to be a SMS of $T$.

1.7. THEOREM. Given $T$, the following assertions are equivalent:

(I) $\{0\}$ is a SMS of T;

(II) for every $Y \in \operatorname{Inv} T$ with $\sigma(T \mid Y)$ compact in $\mathbf{C}$, we have $Y \subset D_{T}$;

(III) for every $Y \in \operatorname{Inv} T, Y \neq\{0\}$ implies that $\sigma(T \mid Y) \neq \varnothing$;

(IV) every T-bounded SMS is a SMS of T.

Proof. (I) $\Rightarrow$ (II). Given $Y \in \operatorname{Inv} T$ with $\sigma(T \mid Y)$ compact in $\mathbf{C}$, Proposition 1.5 gives $Y=\Upsilon \oplus W, \Upsilon \subset D_{T}, \sigma(T \mid \Upsilon)=\sigma(T \mid Y), \sigma(T \mid W)=\varnothing$. Then, by hypothesis, $W=\{0\}$ and hence $Y=\Upsilon \subset D_{T}$.

(II) $\Rightarrow$ (III). Let $Y \in \operatorname{Inv} T$ be such that $Y \neq\{0\}$ and suppose that $\sigma(T \mid Y)=\varnothing . \sigma(T \mid Y)$ being compact in $\mathbf{C}, Y \subset D_{T}$. Hence $T \mid Y$ is bounded and $Y \neq\{0\}$ implies that $\sigma(T \mid Y) \neq \varnothing$. This, however, contradicts the assumption on $\sigma(T \mid Y)$.

(III) $\Rightarrow($ IV): Let $Z$ be a $T$-bounded SMS and let $Y \in \operatorname{Inv} T$ be such that

$$
\sigma(T \mid Y) \subset \sigma(T \mid Z) .
$$

$\sigma(T \mid Z)$ being compact in $\mathbf{C}$, so is $\sigma(T \mid Y)$. It follows from Proposition 1.5 that $Y=\Upsilon \oplus W$ with $\Upsilon, W \in \operatorname{Inv} T, \Upsilon \subset D_{T}, \sigma(T \mid \Upsilon)=\sigma(T \mid Y)$ and

$$
\sigma(T \mid W)=\varnothing .
$$

By hypothesis, (1.6) implies that $W=\{0\}$ and hence

$$
Y=\Upsilon \subset D_{T} \text {. }
$$

It follows from (1.5), (1.7) that $Y \subset Z$ and hence $Z$ is a SMS of $T$.

(IV) $\Rightarrow(\mathrm{I})$. Evidently, $\{0\}$ is a $T$-bounded SMS and hence $\{0\}$ is a SMS of $T$, by hypothesis.

1.8. LEMMA. Let $T$ have the SVEP. If, for $Y \in \operatorname{Inv} T, T \mid Y$ is bounded then $x \in Y$ and $\sigma_{T}(x)=\varnothing$ imply $x=0$. 
Proof. By $\sigma_{T}(x)=\varnothing$, the local resolvent is an entire function. For $x \in Y$, the SVEP implies

$$
x(\lambda)=R(\lambda ; T \mid Y) x, \quad|\lambda|>\|T \mid Y\| .
$$

Consequently, for $\Gamma=\{\lambda \in \mathbf{C}:|\lambda|=\|T \mid Y\|+1\}$, we have

$$
x=\frac{1}{2 \pi i} \int_{\Gamma} R(\lambda ; T \mid Y) x d \lambda=\frac{1}{2 \pi i} \int_{\Gamma} x(\lambda) d \lambda=0 .
$$

1.9. THEOREM. If $T$ has the 1-SDP then, for every compact $F \subset \mathbf{C}$, there exists a T-bounded SMS $\Xi(T, F)$ with the following properties:

(I) $X(T, F)=\Xi(T, F) \oplus X(T, \varnothing)$;

(II) $\sigma[T \mid \Xi(T, F)]=\sigma[T \mid X(T, F)]$.

Proof. Since $\sigma[T \mid X(T, F)] \subset F$ is compact in $\mathbf{C}$, for $Y=X(T, F)$ and $\Upsilon=\Xi(T, F)$, Proposition 1.5 gives

$$
\begin{gathered}
X(T, F)=\Xi(T, F) \oplus W, \\
\sigma[T \mid \Xi(T, F)]=\sigma[T \mid X(T, F)], \quad \sigma(T \mid W)=\varnothing .
\end{gathered}
$$

$X(T, F)$ being a SMS of $T, \Xi(T, F)$ is a $T$-bounded SMS, by Theorem 1.6. Since $X(T, \varnothing)$ is a SMS of $T, \sigma(T \mid W)=\varnothing$ implies $W \subset X(T, \varnothing)$. Conversely, let $x \in X(T, \varnothing)$. Then $x \in X(T, F)$ and hence $Q x \in \Xi(T, F)$, where $Q$ is the projection (1.3) for $Y=X(T, F)$. Since $Q$ commutes with $T$, we have $\sigma_{T}(Q x) \subset \sigma_{T}(x)=\varnothing$ and hence $Q x=0$, by Lemma 1.8. Thus, $X(T, \varnothing) \subset W$ and hence $W=X(T, \varnothing)$.

1.10. Proposition. Given T, every T-bounded SMS and every SMS of $T$ is hyperinvariant under $T$.

Proof. We confine the proof to a $T$-bounded SMS. Let $A \in B(X)$ commute with $T$ and fix $\lambda \in \mathbf{C}$ with $|\lambda|>\|A\|$. Then $R(\lambda ; A)=$ $\sum_{n=0}^{\infty} \lambda^{-n-1} A^{n}$. For every $x \in D_{T}$ and positive integer $k$, we have

$$
\sum_{n=0}^{k} \lambda^{-n-1} A^{n} T x=T\left(\sum_{n=0}^{k} \lambda^{-n-1} A^{n} x\right) .
$$

$T$ being closed, $k \rightarrow \infty$ implies that $R(\lambda ; A) x \in D_{T}$ and

$$
R(\lambda ; A) T x=T R(\lambda ; A) x .
$$


Now, let $Y$ be a $T$-bounded SMS and put $Y_{\lambda}=R(\lambda ; A) Y$. Since $Y \subset D_{T}$, we have $Y_{\lambda} \subset D_{T}$ and $Y_{\lambda} \in$ Inv $T$. For $x \in Y$, (1.8) implies

$$
R(\lambda ; A)^{-1}\left(T \mid Y_{\lambda}\right) R(\lambda ; A) x=(T \mid Y) x,
$$

hence $T \mid Y_{\lambda}$ and $T \mid Y$ are similar. Thus, $\sigma\left(T \mid Y_{\lambda}\right)=\sigma(T \mid Y)$. Since $Y_{\lambda} \subset D_{T}$ and $Y$ is a $T$-bounded SMS, $Y_{\lambda} \subset Y$, i.e. $Y$ is invariant under $R(\lambda ; A)$, for $|\lambda|>\|A\|$. It follows from the identity

$$
A=\lim _{\lambda \rightarrow \infty} \lambda[\lambda R(\lambda ; A)-I],
$$

that $Y$ is invariant under $A$.

1.11. Lemma. Given $T$ with the 1-SDP, let $F \subset \mathbf{C}$ be compact. Then $x \in \Xi(T, F)$ iff

(i) $\sigma_{T}(x) \subset F$ and (ii) $\lim _{\lambda \rightarrow \infty} x(\lambda)=0$.

Proof. (Only if): Let $x \in \Xi(T, F)$. We have

$$
\sigma_{T}(x) \subset \sigma[T \mid \Xi(T, F)]=\sigma[T \mid X(T, F)] \subset F .
$$

Since $T \mid \Xi(T, F)$ is bounded, it follows that

$$
\lim _{\lambda \rightarrow \infty} x(\lambda)=\lim _{\lambda \rightarrow \infty} R[\lambda ; T \mid \Xi(T, F)] x=0 .
$$

(If): By (i), $x \in X(T, F)$. Since $T$ is closed, it follows from (ii) and from the identity

$$
\lambda x(\lambda)-x=T x(\lambda)
$$

that

$$
\lim _{\lambda \rightarrow \infty}[\lambda x(\lambda)-x]=T \lim _{\lambda \rightarrow \infty} x(\lambda)=0 .
$$

The function $f: V \rightarrow X$, defined by $f(\lambda)=\lambda x(\lambda)-x$ is analytic on a neighborhood $V$ of $\infty$ and $f(\infty)=\lim _{\lambda \rightarrow \infty} f(\lambda)=0$. Let $r>0$ be sufficiently large, for

$$
F \subset\{\lambda \in \mathbf{C}:|\lambda|<r\} \text { and } V \supset\{\lambda \in \mathbf{C}:|\lambda| \geq r\} .
$$

We have

$$
x(\lambda)-\frac{x}{\lambda}=\frac{f(\lambda)}{\lambda}
$$


and note that $\infty$ is, at least, a double zero of $f(\lambda) / \lambda$. Consequently, we have

$$
\begin{aligned}
x & =\frac{1}{2 \pi i} \int_{\Gamma} \frac{x}{\lambda} d \lambda=\frac{1}{2 \pi i} \int_{\Gamma} x(\lambda) d \lambda-\frac{1}{2 \pi i} \int_{\Gamma} \frac{f(\lambda)}{\lambda} d \lambda \\
& =\frac{1}{2 \pi i} \int_{\Gamma} R[\lambda ; T \mid X(T, F)] x d \lambda=Q x \in \Xi(T, F),
\end{aligned}
$$

where

$$
\Gamma=\{\lambda \in \mathbf{C}:|\lambda|=r\} \quad \text { and } \quad Q=\frac{1}{2 \pi i} \int_{\Gamma} R[\lambda ; T \mid X(T, F)] d \lambda .
$$

A direct sum decomposition property of $X(T, F)$ for a bounded decomposable operator [1, Lemma 2.3] admits the following generalization.

1.12. TheOrem. Given $T$ with the 1-SDP, let $F_{1} \subset \mathbf{C}$ be closed and $F_{2} \subset \mathbf{C}$ be compact. If $F_{1}$ and $F_{2}$ are disjoint, then

$$
X\left(T, F_{1} \cup F_{2}\right)=X\left(T, F_{1}\right) \oplus \Xi\left(T, F_{2}\right) .
$$

Proof. By denoting $F=F_{1} \cup F_{2}$, one obtain easily

$$
X(T, F) \supset X\left(T, F_{1}\right)+\Xi\left(T, F_{2}\right) .
$$

On the other hand, by the functional calculus $X(T, F)$ admits a direct sum decomposition

$$
X(T, F)=X_{1} \oplus X_{2},
$$

with $\sigma\left(T \mid X_{i}\right) \subset F_{i}(i=1,2)$ and $X_{2} \subset D_{T}$. Then $X_{l} \subset X\left(T, F_{l}\right), i=1,2$. Since $T \mid X_{2}$ is bounded, for every $x \in X_{2}$, we have $\sigma_{T}(x) \subset F_{2}$ and

$$
\lim _{\lambda \rightarrow \infty} x(\lambda)=\lim _{\lambda \rightarrow \infty} R\left(\lambda ; T \mid X_{2}\right) x=0 .
$$

Lemma 1.11 implies that $x \in \Xi\left(T, F_{2}\right)$ and hence $X_{2} \subset \Xi\left(T, F_{2}\right)$. Thus, the opposite of (1.9) is obtained and hence

$$
X(T, F)=X\left(T, F_{1}\right)+\Xi\left(T, F_{2}\right) .
$$

To see that (1.10) is a direct sum, suppose that

$$
x \in X\left(T, F_{1}\right) \cap \Xi\left(T, F_{2}\right) \subset X\left(T, F_{1}\right) \cap X\left(T, F_{2}\right)=X(T, \varnothing) .
$$

Then $\sigma_{T}(x)=\varnothing$ and hence $x(\cdot)$ is an entire function. It follows from $x \in \Xi\left(T, F_{2}\right)$ that $\lim _{\lambda \rightarrow \infty} x(\lambda)=0$ and hence $x(\lambda)=0$. Thus $x=0$. 
1.13. ThEOREM. Given $T$ with the 1-SDP, if $F_{1} \subset \mathrm{C}$ is closed and $F_{2} \subset \mathbf{C}$ is compact then

$$
\Xi\left(T, F_{1} \cap F_{2}\right)=X\left(T, F_{1}\right) \cap \Xi\left(T, F_{2}\right) .
$$

Proof. With the help of Lemma 1.11, inclusion

$$
\Xi\left(T, F_{1} \cap F_{2}\right) \subset X\left(T, F_{1}\right) \cap \Xi\left(T, F_{2}\right)
$$

follows easily. Let $x \in X\left(T, F_{1}\right) \cap \Xi\left(T, F_{2}\right)$. Then $\sigma_{T}(x) \subset F_{1} \cap F_{2}$ and hence $x \in X\left(T, F_{1} \cap F_{2}\right)$. Since $x \in \Xi\left(T, F_{2}\right)$, Lemma 1.11 implies $\lim _{\lambda \rightarrow \infty} x(\lambda)=0$. Quote again Lemma 1.11 and infer that $x \in$ $\Xi\left(T, F_{1} \cap F_{2}\right)$. Thus, the opposite of (1.12) follows and hence (1.11) is obtained.

1.14. Proposition. Given T, if there is a decomposition

$$
X=X_{1}+X_{2} \text { with } X_{1}, X_{2} \in \operatorname{Inv} T \text { and } X_{1} \subset D_{T}
$$

then $T \mid X_{2}$ is densely defined.

Proof. Recall that by our assumption, $T$ is densely defined. For every $x \in X$, there is a representation

$$
x=x_{1}+x_{2}, \quad x_{i} \in X_{i}, i=1,2,
$$

and there is a number $M>0$ (independent of $x$ ) such that $\left\|x_{1}\right\|+\left\|x_{2}\right\| \leq$ $M\|x\|$. Let $x \in X_{2}$. There is a sequence $\left\{x_{n}\right\} \subset D_{T}$ converging to $x$. For every $n$, there is a representation

$$
x-x_{n}=x_{n 1}+x_{n 2}, \quad x_{n l} \in X_{l}(i=1,2)
$$

with

$$
\left\|x_{n 1}\right\|+\left\|x_{n 2}\right\| \leq M\left\|x-x_{n}\right\| .
$$

Then $x_{n l} \rightarrow 0(i=1,2)$ as $n \rightarrow \infty$. By hypothesis,

$$
y_{n}=x-x_{n 2}=x_{n}+x_{n 1} \in D_{T} \cap X_{2}
$$

and hence

$$
\left\|x-y_{n}\right\|=\left\|x_{n 2}\right\| \rightarrow 0 \quad \text { as } n \rightarrow \infty
$$

implies that $T \mid X_{2}$ is densely defined.

1.15. Lemma. Given $T$ and $Y \in \operatorname{Inv} T$, consider the following conditions:

$$
\sigma(T) \cup \sigma(T \mid Y) \neq \mathbf{C} ;
$$




$$
\hat{T}=T / Y \text { is a closed operator on } X / Y \text {. }
$$

Then (1.13) implies (1.14) and either of them implies inclusions

$$
\begin{gathered}
\sigma(\hat{T}) \subset \sigma(T \mid Y) \cup \sigma(T) ; \quad \sigma(T \mid Y) \subset \sigma(T) \cup \sigma(\hat{T}) ; \\
\sigma(T) \subset \sigma(\hat{T}) \cup \sigma(T \mid Y) .
\end{gathered}
$$

Proof. Assume (1.13) and let $\lambda \in \rho(T) \cap \rho(T \mid Y)$ be arbitrary. For every $x \in Y$, we have $R(\lambda ; T) x=R(\lambda ; T \mid Y) x \in Y$ and hence $Y$ is invariant under $R(\lambda ; T)$. Denote $R_{\lambda}=R(\lambda ; T)$ and let $\hat{R}_{\lambda}$ be the coinduced operator by $R_{\lambda}$ on the quotient space $X / Y$. The identities

$$
(\lambda-T) R_{\lambda} x=x, \quad x \in X ; \quad R_{\lambda}(\lambda-T) x=x, \quad x \in D_{T},
$$

give rise to

(1.16) $(\lambda-\hat{T}) \hat{R}_{\lambda} \hat{x}=\hat{x}, \quad \hat{x} \in X / Y ; \quad \hat{R}_{\lambda}(\lambda-\hat{T}) \hat{x}=\hat{x}, \quad \hat{x} \in D_{\hat{T}}$.

It follows from (1.16) that $\hat{R}_{\lambda}$ is the inverse of $\lambda-\hat{T}$. Since $\hat{R}_{\lambda}$ is bounded and defined on $X / Y$, it is closed and hence $\hat{T}$ is closed. By (1.16), $\lambda \in \rho(\hat{T})$ and this implies the first of (1.15). The remainder of the proof is routine and we omit it (see [2, Proposition 2.2]).

1.16. Lemma. Given $T$, let $X_{0}, X_{1}, Y \in \operatorname{Inv} T$ satisfy the following conditions:

$$
\begin{aligned}
X=X_{0}+X_{1}, \quad X_{1} & \subset D_{T} \cap Y ; \\
\sigma\left(T \mid X_{0}\right) & \subset F, \quad \sigma\left(T \mid X_{0} \cap Y\right) \subset F,
\end{aligned}
$$$$
\text { for some closed } F \subset \mathbf{C}, F \neq \mathbf{C} \text {. }
$$

Then $\hat{T}=T / Y$ is closed on $X / Y$. Furthermore, if $\tilde{T}=\left(T \mid X_{0}\right) / Y \cap X_{0}$, (i.e. $\tilde{T}$ is the coinduced operator by $T \mid X_{0}$ on the quotient space $X_{0} / Y \cap X_{0}$ ), then

$$
\sigma(\hat{T})=\sigma(\tilde{T})
$$

Proof. The quotient spaces $X / Y$ and $X_{0} / Y \cap X_{0}$ are topologically isomorphic. Since by (1.18), $\sigma\left(T \mid X_{0}\right) \cup \sigma\left(T \mid Y \cap X_{0}\right) \neq \mathbf{C}$, Lemma 1.15 implies that $\tilde{T}$ is closed.

Next, we show that $\tilde{T}$ and $\hat{T}$ are similar. In view of (1.17), every $x \in D_{T}$ has a representation

$$
x=x_{0}+x_{1} \quad \text { with } x_{i} \in X_{i}, \quad i=0,1 .
$$


Since $x_{1} \in D_{T}$, we have $x_{0} \in D_{T}$. Thus, $x_{1} \in Y \cap D_{T}$ and $x_{0} \in X_{0} \cap D_{T}$. Let $A=X / Y \rightarrow X_{0} / Y \cap X_{0}$ be the topological isomorphism. For $x \in X$, let $\hat{x}=x+Y \in X / Y$ and, for $x \in X_{0}, \tilde{x}=x+Y \cap X_{0} \in X_{0} / Y \cap X_{0}$. For every $\hat{x} \in D_{\hat{T}}$, there is $x \in \hat{x} \cap D_{T}$ and we have $A \hat{x}=A \hat{x}_{0}=\tilde{x}_{0} \in D_{\tilde{T}}$. Conversely, for every $\tilde{x} \in D_{\tilde{T}}$, there is $x \in \tilde{x} \cap\left(X_{0} \cap D_{T}\right)$ and hence $\hat{x} \in D_{\hat{T}}$. Consequently, $A D_{\hat{T}}=D_{\tilde{T}}$. For every $\hat{x} \in D_{\hat{T}}$, we obtain successively

$$
A \hat{T} \hat{x}=A(T x)^{\hat{x}}=(T x)^{\tilde{y}}=\tilde{T} \tilde{x}=\tilde{T} A \hat{x}
$$

and hence $\hat{T}$ is similar to $\tilde{T}$. Therefore, $\hat{T}$ is closed and (1.19) holds.

1.17. ThEOREM. Given $T$ with the $1-S D P$, let $G \subset \mathbf{C}$ be open and put

$$
Y= \begin{cases}X(T, \bar{G}), & \text { if } G \text { is unbounded } \\ \Xi(T, \bar{G}), & \text { if } G \text { is bounded. }\end{cases}
$$

Then $\hat{T}=T / Y$ is closed and

$$
\sigma(\hat{T}) \subset G^{c} \text {. }
$$

In particular, if $G \in V_{\infty}$, then $\hat{T}$ is bounded.

Proof. First, suppose that $G \notin V_{\infty}$. Let $\lambda \in G$ be arbitrary and let $\left\{G_{0}, G_{1}\right\} \in \operatorname{cov} \sigma(T)$ satisfy conditions: $G_{0} \in V_{\infty}, \lambda \notin \bar{G}_{0}, \lambda \in G_{1} \subset \bar{G}_{1}$ $\subset G$ and $G_{1}$ is relatively compact in C. By the 1-SDP,

$$
X=X\left(T, \bar{G}_{0}\right)+X\left(T, \bar{G}_{1}\right)
$$

and since $X(T, \varnothing) \subset X\left(T, \bar{G}_{0}\right)$, Theorem 1.9 implies the spectral decomposition

$$
X=X\left(T, \bar{G}_{0}\right)+\Xi\left(T, \bar{G}_{1}\right) .
$$

Put $X_{0}=X\left(T, \bar{G}_{0}\right), X_{1}=\Xi\left(T, \bar{G}_{1}\right)$ and obtain

$$
X_{1} \subset D_{T} \cap Y \text {. }
$$

Furthermore, we have

$$
\begin{gathered}
\sigma\left(T \mid X_{0}\right)=\sigma\left[T \mid X\left(T, \bar{G}_{0}\right)\right] \subset \bar{G}_{0} \\
\sigma\left[T \mid X\left(T, \bar{G}_{0}\right) \cap X(T, \bar{G})\right]=\sigma\left[T \mid X\left(T, \bar{G}_{0} \cap \bar{G}\right)\right] \subset \bar{G}_{0} \\
\sigma\left[T \mid X\left(T, \bar{G}_{0}\right) \cap \Xi(T, \bar{G})\right]=\sigma\left[T \mid \Xi\left(T, \bar{G}_{0} \cap \bar{G}\right)\right] \subset \bar{G}_{0},
\end{gathered}
$$

The last equality in (1.25) stems from Theorem 1.13. In view of the definition of $Y$, relations (1.21)-(1.25) fulfill all hypotheses of Lemma 
1.16. Thus, $\hat{T}$ is closed and

$$
\sigma(\hat{T})=\sigma(\tilde{T}),
$$

where $\tilde{T}=\left(T \mid X_{0}\right) / Y \cap X_{0}$. It follows from Lemma 1.15 and from (1.23)-(1.25) that

$$
\sigma(\tilde{T}) \subset \sigma\left(T \mid X_{0}\right) \cup \sigma\left(T \mid X_{0} \cap Y\right) \subset \bar{G}_{0} .
$$

Since $\lambda \notin \bar{G}_{0},(1.26)$ and (1.27) imply that $\lambda \notin \sigma(\hat{T})$. Thus, (1.20) follows.

For $G \in V_{\infty}$, we may assume that $\mathbf{C} \neq \bar{G}$. Let $\lambda \in G$ be arbitrary. Choose $G_{1}$ relatively compact in $\mathbf{C}$ such that $\left\{G, G_{1}\right\} \in \operatorname{cov} \sigma(T)$ and $\lambda \notin \bar{G}_{1}$. Since $\hat{T}$ and $\tilde{T}=\left[T \mid \Xi\left(T, \bar{G}_{1}\right)\right] / \Xi\left(T, \bar{G}_{1}\right) \cap Y$ are similar and $\tilde{T}$ is bounded, $\sigma(\tilde{T})=\sigma(\hat{T})$ and $\hat{T}$ is bounded. By Lemma 1.15,

$$
\sigma(\hat{T})=\sigma(\tilde{T}) \subset \sigma\left[T \mid \Xi\left(T, \bar{G}_{1}\right)\right] \cup \sigma\left[T \mid \Xi\left(T, \bar{G} \cap \bar{G}_{1}\right)\right] \subset \bar{G}_{1}
$$

and hence $\lambda \in \rho(\hat{T})$. Thus, inclusion (1.20) follows.

While the two-summand spectral decomposition property (1-SDP) of the given operator is a convenient mechanism in our spectral theoretic study, it does not confine its scope. Similarly to some other types of spectral decompositions (i.e. [6,7]) it is shown that the 1-SDP and the general SDP are equivalent. Details of that proof will be included in another work. The two extensions of the spectral maximal space concept give rise to two generalizations of the decomposable operator concept.

1.18. Definition. $T$ is said to be decomposable if, for any $\left\{G_{l}\right\}_{l=0}^{n} \in$ $\operatorname{cov} \sigma(T)$ with $G_{0} \in V_{\infty}$, there is a system $\left\{Y_{i}\right\}_{i=0}^{n}$ of SMS of $T$ satisfying conditions (I) and (II) of Definition 1.1.

1.19. Definition. $T$ is said to be $\{\infty\}$-decomposable if, for any $\left\{G_{l}\right\}_{l=0}^{n} \in \operatorname{cov} \sigma(T)$ with $G_{0} \in V_{\infty}$, there is a SMS $Y_{0}$ of $T$ and a system $\left\{Y_{i}\right\}_{i=1}^{n}$ of $T$-bounded SMS satisfying conditions (II) of Definition 1.1.

The case of $\{\infty\}$-decomposable operator fits into the theory of the residually decomposable operators $[8,9]$ with residuum $S=\{\infty\}$. If $T$ is $\{\infty\}$-decomposable for $n$ confined to $n=1$, then $T$ is said to be $(\{\infty\}, 1)$-decomposable. If $T$ is $(\{\infty\}, 1)$-decomposable then its conjugate $T^{*}$ is again $(\{\infty\}, 1)$-decomposable [9]. Moreover, for every open $G \subset \mathbf{C}$, the spectral manifold $X(T, \bar{G})$ is closed in $X$, as a fulfilment of condition $\gamma$ [ibid.].

We conclude this section with some necessary and sufficient conditions which make the unbounded operators with the SDP and the unbounded decomposable operators equivalent. 
1.20. THEOREM. Given T, the following assertions are equivalent:

(I) $T$ is decomposable;

(II) $T$ has the $S D P$ and $X(T, \varnothing)=\{0\}$, or

$T$ has the SDP and $\{0\}$ is a SMS of T;

(III) $T$ has the SDP and every T-bounded SMS is a SMS of T;

(IV) $T$ has the $S D P$ and $X(T, F) \subset D_{T}$ for some compact $F$ in $\mathbf{C}$.

Proof. The conclusion will be reached through the following sequel of implications: (I) $\Rightarrow$ (II) $\Rightarrow$ (III) $\Rightarrow$ (IV) $\Rightarrow$ (II) and (III) $\Rightarrow$ (I).

(I) $\Rightarrow$ (II). Clearly, $T$ has the SDP. Let $\left\{G_{0}, G_{1}\right\} \in \operatorname{cov} \sigma(T)$ with $G_{0} \in V_{\infty}$. There corresponds the spectral decomposition

$$
X=X_{0}+X_{1} \text { with } X_{1} \subset D_{T} \text { spectral maximal. }
$$

Consequently, $\sigma[T \mid X(T, \varnothing)]=\varnothing \subset \sigma\left(T \mid X_{1}\right)$ implies $X(T, \varnothing) \subset X_{1} \subset$ $D_{T}$. Then $X(T, \varnothing)=\{0\}$, by Lemma 1.8.

(II) $\Rightarrow$ (III) follows from Theorem 1.7.

(III) $\Rightarrow$ (IV). Let $F \subset \mathbf{C}$ be compact. By hypothesis, $\Xi(T, F)$ is a SMS of $T$. Then $\sigma[T \mid X(T, F)]=\sigma[T \mid \Xi(T, F)]$ implies $X(T, F) \subset \Xi(T, F) \subset$ $D_{T}$.

$$
\begin{gathered}
\text { (IV) } \Rightarrow \text { (II). If, for some compact } F \subset \mathbf{C}, X(T, F) \subset D_{T}, \text { then } \\
X(T, F)=\Xi(T, F) \oplus X(T, \varnothing) \text { and } \Xi(T, F) \subset D_{T}
\end{gathered}
$$

imply that $X(T, \varnothing)=\{0\}$.

$(\mathrm{III}) \Rightarrow(\mathrm{I})$. By the SDP, for any $\left\{G_{i}\right\}_{i=0}^{n} \in \operatorname{cov} \sigma(T)$ with $G_{0} \in V_{\infty}$, there exists $\left\{Y_{l}\right\}_{l=0}^{n} \subset \operatorname{Inv} T$ such that $\sigma\left(T \mid Y_{i}\right) \subset G_{l}(0 \leq i \leq n)$ and

$$
X=\sum_{l=0}^{n} Y_{l} \subset \sum_{l=0}^{n} X\left(T, \bar{G}_{l}\right) \subset X .
$$

By Proposition 1.4, every $X\left(T, \bar{G}_{l}\right)$ is a SMS of $T$. Moreover,

$$
X\left(T, \bar{G}_{l}\right)=\Xi\left(T, \bar{G}_{i}\right) \oplus X(T, \varnothing), \quad 1 \leq i \leq n, \quad X(T, \varnothing) \subset X\left(T, \bar{G}_{0}\right)
$$

imply

$$
X=X\left(T, \bar{G}_{0}\right)+\sum_{i=1}^{n} \Xi\left(T, \bar{G}_{l}\right) .
$$

Since $X\left(T, \bar{G}_{0}\right)$ and, by hypothesis, every $\Xi\left(T, \bar{G}_{t}\right)(1 \leq i \leq n)$ is a SMS of $T, T$ is decomposable.

(After this paper was accepted for publication, we noticed that Lemma 1.15 appeared explicitly in F.-H. Vasilescu, Analytic Functional Calculus and Spectral Decompositions, D. Reidel, Dordrecht: Holland, 1982.) 
2. Elements of spectral duality theory. While this section prepares the main Theorem 3.1, some of the properties discussed here have intrinsic values. Various topologies are involved in the duality theory. If $A$ and $B$ are dual spaces, we use the notation $\tau(A, B)$ for the topology on $A$ induced by $B$, under the given duality.

\subsection{THEOREM. If $T$ has the SDP then $T^{*}$ has the SDP.}

Proof. Let $T$ have the SDP. Then, for closed $F \subset \mathbf{C}, X(T, F)$ is closed (Proposition 1.4). Let $\left\{G_{0}, G_{1}\right\} \in \operatorname{cov} \sigma(T)$ with $G_{0} \in V_{\infty}$. The SDP implies the spectral decomposition (1.21)

$$
X=X\left(T, \bar{G}_{0}\right)+\Xi\left(T, \bar{G}_{1}\right) .
$$

Since $X\left(T, \bar{G}_{0}\right)$ is a SMS of $T$ and $\Xi\left(T, \bar{G}_{1}\right)$ is a $T$-bounded SMS, $T$ is a $(\{\infty\}, 1)$-decomposable operator. By [9, Theorem 2.10] as mentioned in 1, $T^{*}$ is $(\{\infty\}, 1)$-decomposable. Consequently, $T^{*}$ has the 1-SDP and hence it has the SDP.

2.2. Lemma. Given $T$, let $Y \in \operatorname{Inv} T$ be such that $Y \subset D_{T}$. If $T^{*} \mid Y^{\perp}$ is densely defined then $T / Y$ is closable. Moreover, $(T / Y)^{*}=T^{*} \mid Y^{\perp}$.

Proof. $Y^{\perp}$ can be viewed as the conjugate of $X / Y$, under the isometric isomorphism $(X / Y)^{*} \rightarrow Y^{\perp}$. For convenience, we make no distinction between $Y^{\perp}$ and $(X / Y)^{*}$ and denote by $\left\langle\hat{x}, x^{*}\right\rangle$ the linear functional $x^{*} \in Y^{\perp}$ on $X / Y$. For $\hat{x} \in D_{T / Y}, x \in \hat{x} \cap D_{T}, y \in Y$ and $x^{*} \in Y^{\perp} \cap D_{T^{*}}$, we have

$$
\begin{aligned}
\left\langle(T / Y) \hat{x}, x^{*}\right\rangle & =\left\langle T(x+y), x^{*}\right\rangle=\left\langle T x, x^{*}\right\rangle \\
& =\left\langle x, T^{*} x^{*}\right\rangle=\left\langle x+y, T^{*} x^{*}\right\rangle=\left\langle\hat{x}, T^{*} x^{*}\right\rangle .
\end{aligned}
$$

Hence $T / Y$ and $T^{*} \mid Y^{\perp}$ are conjugates to each other. Since $T^{*} \mid Y^{\perp}$ is densely defined, $T / Y$ is closable (e.g. [4, III. Theorem 5.28]). It remains to prove the second statement of the lemma. Let $\overline{T / Y}$ be the minimal closed extension of $T / Y$. It follows from $\bar{D}_{T}=X$ that $\bar{D}_{T / Y}=X / Y$ and hence $(\overline{T / Y})^{*}=(T / Y)^{*}$ exists. Then (2.1) implies

$$
G^{\prime}\left(-T^{*} \mid Y^{\perp}\right) \subset[G(T / Y)]^{\perp}=G^{\prime}\left[-(T / Y)^{*}\right] .
$$

It follows from (2.2) that $(T / Y)^{*} \supset T^{*} \mid Y^{\perp}$.

To prove the opposite inclusion, let $x^{*} \in D_{(T / Y)^{*}}$. For $x \in D_{T}$ and $y \in Y$, we obtain successively

$$
\begin{aligned}
\left\langle(\overline{T / Y}) \hat{x}, x^{*}\right\rangle & =\left\langle\hat{x},(T / Y)^{*} x^{*}\right\rangle \\
& =\left\langle x+y,(T / Y)^{*} x^{*}\right\rangle=\left\langle x,(T / Y)^{*} x^{*}\right\rangle .
\end{aligned}
$$


Thus, for every $x^{*} \in D_{(T / Y)^{*}},\left\langle(\overline{T / Y}) \hat{x}, x^{*}\right\rangle$ is a bounded linear functional of $x$ and hence $x^{*} \in D_{T^{*}}$. Furthermore, $x^{*} \in D_{(T / Y)^{*}} \subset Y^{\perp}$ and hence $x^{*} \in Y^{\perp} \cap D_{T^{*}}$.

On the other hand, since $x \in D_{T}$, for every $y \in Y$, we have

$$
\begin{aligned}
\left\langle(\overline{T / Y}) \hat{x}, x^{*}\right\rangle & =\left\langle(T / Y) \hat{x}, x^{*}\right\rangle \\
& =\left\langle T(x+y), x^{*}\right\rangle=\left\langle T x, x^{*}\right\rangle=\left\langle x, T^{*} x^{*}\right\rangle .
\end{aligned}
$$

It follows from (2.3) and (2.4) that $(T / Y)^{*} \subset T^{*} \mid Y^{\perp}$.

2.3. LEMMA. Suppose that $Y^{* * *} \subset X^{* * *}$ is closed for $\tau\left(X^{* * *}, X^{* *}\right)$ and $Y^{* * *}$ is invariant under the projection $P$ of $X^{* * *}$ onto $K X^{*}$, along $(J X)^{\perp}$. Then $P Y^{* * *}$ is closed for $\tau\left(K X^{*}, J X\right)$.

Proof. Let $S^{* * *}$ be the closed (for the metric topology of $X^{* * *}$ ) unit ball of $P Y^{* * *}$. Let $\left\{x_{\alpha}^{* * *}\right\} \subset S^{* * *}$ be a net converging to $x_{0}^{* * *} \in K X^{*}$ for $\tau\left(K X^{*}, J X\right)$. Since $\left\{x_{\alpha}^{* * *}\right\}$ is bounded in $X^{* * *}$, there is a subnet $\left\{x_{\beta}^{* * *}\right\}$ of $\left\{x_{\alpha}^{* * *}\right\}$ such that $x_{\beta}^{* * *} \rightarrow x^{* * *} \in X^{* * *}$ for $\tau\left(X^{* * *}, X^{* *}\right)$. Since, by hypothesis, $Y^{* * *}$ is closed for $\tau\left(X^{* * *}, X^{* *}\right)$, we have $x^{* * *} \in$ $Y^{* * *}$. Let $J x \in J X$. Then $\left\langle J x,(I-P) x^{* * *}\right\rangle=0$ and hence

$$
\lim _{\beta}\left\langle J x, x_{\beta}^{* * *}\right\rangle=\left\langle J x, x^{* * *}\right\rangle=\left\langle J x, P x^{* * *}\right\rangle .
$$

On the other hand, we have

$$
\lim _{\beta}\left\langle J x, x_{\beta}^{* * *}\right\rangle=\lim _{\alpha}\left\langle J x, x_{\alpha}^{* * *}\right\rangle=\left\langle J x, x_{0}^{* * *}\right\rangle .
$$

It follows from (2.5) and (2.6) that

$$
\left\langle J x, P x^{* * *}\right\rangle=\left\langle J x, x_{0}^{* * *}\right\rangle .
$$

Since both $P x^{* * *}$ and $x_{0}^{* * *}$ are elements of $K X^{*}$, we have

$$
x_{0}^{* * *}=P x^{* * *} \in P Y^{* * *} .
$$

Since, clearly $\left\|x_{0}^{* * *}\right\| \leq 1,(2.7)$ implies that $x_{0}^{* * *} \in S^{* * *}$ and hence $S^{* * *}$ is closed for $\tau\left(K X^{*}, J X\right)$. By the Krein-S̆Smul'jan theorem, $P Y^{* * *}$ is closed for $\tau\left(K X^{*}, J X\right)$.

2.4. THEOREM. Given $T$, the following properties hold:

(i) If the density condition (*) is satisfied then, for every $x \in D_{T}$, we have $J x \in D_{T^{* *}}$ and $T^{* *} J x=J T x$; likewise

(i') if the density condition (**) is satisfied then, for every $x^{*} \in D_{T^{*}}$, we have $K x^{*} \in D_{T^{* * *}}$ and $T^{* * *} K x^{*}=K T^{*} x^{*}$.

(ii) Suppose that the density condition $(* *)$ is satisfied and $x^{* * *} \in K X^{*}$. If $\left\langle T^{* *} J x, x^{* * *}\right\rangle$ is a bounded linear functional of $J x \in J D_{T}$, then $x^{* * *} \in$ $K D_{T^{*}}$ and $T^{* * *} x^{* * *}=K T^{*} K^{-1} x^{* * *}$. 
Proof. (i): Since $G(t)={ }^{\perp}\left[G^{\prime}\left(-T^{*}\right)\right]$ and $\left[G^{\prime}\left(-T^{*}\right)\right]^{\perp}=G\left(T^{* *}\right)$, for every $(x, T x) \in G(T)$ we have $(J x, J T x) \in G\left(T^{* *}\right)$ or, equivalently, $J x$ $\in D_{T^{* *}}$ and $T^{* *} J x=J T x$.

(i') follows directly from (i), with the original space $X^{*}$ and embed$\operatorname{ding} K$.

(ii): Let $x \in D_{T}$ and suppose that $\left\langle T^{* *} J x, x^{* * *}\right\rangle$ is a bounded linear functional of $J x$. With the help of (i), we obtain

$$
\begin{aligned}
\left\langle T x, K^{-1} x^{* * *}\right\rangle & =\left\langle K^{-1} x^{* * *}, J T x\right\rangle=\left\langle K^{-1} x^{* * *}, T^{* *} J x\right\rangle \\
& =\left\langle T^{* *} J x, x^{* * *}\right\rangle
\end{aligned}
$$

and hence $K^{-1} x^{* * *}$ is a bounded linear functional of $x$. Then $K^{-1} x^{* * *} \in$ $D_{T^{*}}$ and by (i'), we obtain $x^{* * *} \in D_{T^{* * *}}$ and $T^{* * *} x^{* * *}=K T^{*} K^{-1} x^{* * *} \cdot \square$

2.5. Corollary. Given T, suppose that (**) holds. Then

$$
K D_{T^{*}}=K X^{*} \cap D_{T^{* * *}} \text {. }
$$

Proof. It follows from Theorem 2.4 (i') that $K D_{T^{*}} \subset K X^{*} \cap D_{T^{* * *}}$. To obtain the opposite inclusion, let $x^{* * *} \in K X^{*} \cap D_{T^{* * *}}$. Then

$$
\left\langle T^{* *} J x, x^{* * *}\right\rangle=\left\langle J x, T^{* * *} x^{* * *}\right\rangle
$$

is a bounded linear functional of $J x$. By Theorem 2.4 (ii), $x^{* * *} \in K D_{T^{*}}$ and hence $K X^{*} \cap D_{T^{* * *}} \subset K D_{T^{*}}$.

2.6. Lemma. Given $T$, assume that (**) holds. Then, the projection $P$ of $X^{* * *}$ onto $K X^{*}$, along $(J X)^{\perp}$ commutes with $T^{* * *}$.

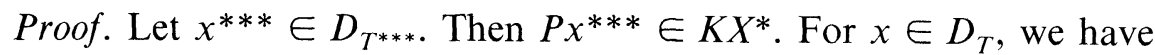
successively

$$
\begin{aligned}
\left\langle T^{* *} J x, P x^{* * *}\right\rangle & =\left\langle T^{* *} J x, P x^{* * *}\right\rangle+\left\langle T^{* *} J x,(I-P) x^{* * *}\right\rangle \\
& =\left\langle T^{* *} J x, x^{* * *}\right\rangle=\left\langle J x, T^{* * *} x^{* * *}\right\rangle=\left\langle J x, P T^{* * *} x^{* * *}\right\rangle
\end{aligned}
$$

and hence $\left\langle T^{* *} J x, P x^{* * *}\right\rangle$ is a bounded linear functional of $J x \in J D_{T}$. Theorem 2.4 (ii) implies that $P x^{* * *} \in D_{T^{* * *}}$. Then

$$
\left\langle T^{* *} J x, P x^{* * *}\right\rangle=\left\langle J x, T^{* * *} P x^{* * *}\right\rangle
$$

implies that

$$
T^{* * *} P x^{* * *}=P T^{* * *} x^{* * *} .
$$


2.7. THEOREM. Given T, assume that (***) is satisfied. Then

$$
J D_{T}=J X \cap D_{T^{* *}} .
$$

Moreover, for every $J x \in J D_{T}$, we have

$$
J T x=T^{* *} J x .
$$

Proof. In view of Theorem 2.4 (i), $J D_{T} \subset J X \cap D_{T^{* *}}$. Let $x^{* * *} \in$ $(J X)^{\perp}$. There is a sequence $\left\{x_{n}^{* * *}\right\} \subset D_{T^{* * *}}$ such that $x_{n}^{* * *} \rightarrow x^{* * *}$ as $n \rightarrow \infty$. By Lemma 2.6, for every $n, P x_{n}^{* * *} \in D_{T^{* * *}}$ and hence $(I-P) x_{n}^{* * *} \in D_{T^{* * *}}$. Thus,

$$
(I-P) x_{n}^{* * *} \rightarrow(I-P) x^{* * *}=x^{* * *} .
$$

Consequently, $(J X)^{\perp} \cap D_{T^{* * *}}$ is dense in $(J X)^{\perp}$.

Now let $J x \in J X \cap D_{T^{* *}}$. For every $x^{* * *} \in(J X)^{\perp} \cap D_{T^{* * *}}$, we have

$$
0=\left\langle J x, T^{* * *} x^{* * *}\right\rangle=\left\langle T^{* *} J x, x^{* * *}\right\rangle .
$$

Since $(J X)^{\perp} \cap D_{T^{* * *}}$ is dense in $(J X)^{\perp}$, it follows from (2.8) that $T^{* *} J x$ $\in J X$. For $x^{*} \in D_{T^{*}}$, Theorem $2.4\left(\mathrm{i}^{\prime}\right)$ implies that

$$
T^{* * *} K x^{*}=K T^{*} x^{*}
$$

and hence, we obtain successively

$$
\begin{aligned}
\left\langle x, T^{*} x^{*}\right\rangle & =\left\langle J x, K T^{*} x^{*}\right\rangle=\left\langle J x, T^{* * *} K x^{*}\right\rangle=\left\langle T^{* *} J x, K x^{*}\right\rangle \\
& =\left\langle x^{*}, T^{* *} J x\right\rangle=\left\langle J^{-1} T^{* *} J x, x^{*}\right\rangle .
\end{aligned}
$$

This means that the element $\left(x, J^{-1} T^{* *} J x\right) \in^{\perp}\left[G^{\prime}\left(-T^{*}\right)\right]=G(T)$. Consequently, $x \in D_{T}$ and $J^{-1} T^{* *} J x=T x$, i.e. $T^{* *} J x=J T x$. Thus, it follows that

$$
J X \cap D_{T^{* *}} \subset J D_{T}
$$

2.8. TheOREM. Given $T$, assume that condition (**) holds. If $T^{*}$ has the SDP then

(i) for every closed $F \subset \mathbf{C}, X^{*}\left(T^{*}, F\right)$ is closed for $\tau\left(X^{*}, X\right)$;

(ii) for every compact $F \subset \mathbf{C}, \Xi^{*}\left(T^{*}, F\right)$ is closed for $\tau\left(X^{*}, X\right)$.

Proof. We confine the proof to (ii), that of (i) is similar. Assuming that $T^{*}$ has the SDP, it follows from Theorem 2.1 that both $T^{* *}$ and $T^{* * *}$ have the SDP. Consequently, $\Xi^{* * *}\left(T^{* * *}, F\right)$ is a $T^{* * *}$-bounded SMS and it is closed for $\tau\left(K X^{*}, J X\right)$, by [9, Proposition 2.9]. It follows from Lemma 2.6, Proposition 1.10, and Lemma 2.3 that $P \Xi^{* * *}\left(T^{* * *}, F\right)$ is closed for $\tau\left(K X^{*}, J X\right)$. 
Next, we prove the equality

$$
K \Xi^{*}\left(T^{*}, F\right)=P \Xi^{* * *}\left(T^{* * *}, F\right) .
$$

Let $x^{* * *} \in K^{* *}\left(T^{*}, F\right)$. Since $T^{*}$ and $T^{* * *} \mid K X^{*}$ are similar (Theorem 2.4 (ii)), $T^{* * *} \mid K X^{*}$ has the SDP and $K \Xi^{*}\left(T^{*}, F\right)$ is a $T^{* * *} \mid K X^{*}$-bounded SMS. Consequently, $\sigma_{T^{* * *}}\left(x^{* * *}\right) \subset F$ and $\lim _{\lambda \rightarrow \infty} x^{* * *}(\lambda)=0$. Lemma 1.11 implies that $x^{* * *} \in \Xi^{* * *}\left(T^{* * *}, F\right)$ and hence

$$
K \Xi^{*}\left(T^{*}, F\right)=P K \Xi^{*}\left(T^{*}, F\right) \subset P \Xi^{* * *}\left(T^{* * *}, F\right) .
$$

Conversely, let $x^{* * *} \in \Xi^{* * *}\left(T^{* * *}, F\right)$. Then Lemma 1.11 implies that $\sigma_{T^{* * *}}\left(x^{* * *}\right) \subset F$ and $\lim _{\lambda \rightarrow \infty} x^{* * *}(\lambda)=0$. Since, by Lemma 2.6 and Proposition 1.10, $P^{* * *}\left(T^{* * *}, F\right) \subset \Xi^{* * *}\left(T^{* * *}, F\right)$, we have

$$
\sigma_{T^{* * *}}\left(P x^{* * *}\right) \subset F \text { and } \lim _{\lambda \rightarrow \infty} P x^{* * *}(\lambda)=0 .
$$

Since $x^{* * *}(\lambda) \in D_{T^{* * *}}$, it follows from Lemma 2.6 that $P x^{* * *}(\lambda) \in$ $D_{T^{* * *}}$. By Corollary 2.5, $P x^{* * *}(\lambda) \in K D_{T^{*}}$ and hence, for $\lambda \in$ $\rho_{T^{* * *}}\left(x^{* * *}\right)$, we obtain

$$
\begin{aligned}
\left(\lambda-T^{*}\right) K^{-1} P x^{* * *}(\lambda) & =K^{-1}\left(\lambda-T^{* * *}\right) P x^{* * *}(\lambda) \\
& =K^{-1} P\left(\lambda-T^{* * *}\right) x^{* * *}(\lambda)=K^{-1} P x^{* * *} .
\end{aligned}
$$

Thus, $\sigma_{T^{*}}\left(K^{-1} P x^{* * *}\right) \subset F$. Since $\lim _{\lambda \rightarrow \infty} K^{-1} P x^{* * *}(\lambda)=0$, Lemma 1.11 implies that $K^{-1} P x^{* * *} \in \Xi^{*}\left(T^{*}, F\right)$, i.e. $P x^{* * *} \in K \Xi^{*}\left(T^{*}, F\right)$. Thus, we have

$$
P \Xi * * *\left(T^{* * *}, F\right) \subset K \Xi *\left(T^{*}, F\right)
$$

and hence (2.9) follows from (2.10) and (2.11). Now, by Lemma 2.3, $K \Xi^{*}\left(T^{*}, F\right)$ is closed for $\tau\left(K X^{*}, J X\right)$ and hence $\Xi^{*}\left(T^{*}, F\right)$ is closed for $\tau\left(X^{*}, X\right)$.

2.9. Theorem. Given $T$, assume that condition $(* * *)$ holds and $T^{*}$ has the SDP. Let $G \subset \mathbf{C}$ be open, $G \in V_{\infty}$ and $Y={ }^{\perp} X^{*}\left(T^{*}, \bar{G}\right)$. Then

(I) $Y \subset D_{T}, Y \in \operatorname{Inv} T, \sigma(T \mid Y) \subset G^{c}$;

(II) $T / Y$ is closable and, for its minimal closed extension $\overline{T / Y}$, we have $\sigma(\overline{T / Y}) \subset \bar{G}$.

Proof. Since $T^{*}$ has the SDP, $X^{*}\left(T^{*}, \bar{G}\right)$ is closed for $\tau\left(X^{*}, X\right)$ and hence $Y^{\perp}=X^{*}\left(T^{*}, \bar{G}\right)$. Clearly, $X^{*}\left(T^{*}, \bar{G}\right)$ is invariant under $T^{*}$. Let $H$ be a relatively compact open set such that $G^{c} \subset H$. Then $\{G, H\} \in \operatorname{cov} \mathbf{C}$ with $G \in V_{\infty}$ and the SDP of $T^{*}$ gives rise to the decomposition

$$
X^{*}=X^{*}\left(T^{*}, \bar{G}\right)+\Xi^{*}\left(T^{*}, \bar{H}\right) .
$$


Since $\Xi^{*}\left(T^{*}, \bar{H}\right) \subset D_{T^{*}}$, it follows from Proposition 1.14 that

$$
T^{*}\left|X^{*}\left(T^{*}, \bar{G}\right)=T^{*}\right| Y^{\perp}
$$

is densely defined. It can be easily shown that $Y \in \operatorname{Inv} T$.

Next, we show that $Y \subset D_{T}$. In view of (2.12), for every $x^{*} \in X^{*}$, there is a representation

$$
x^{*}=x_{1}^{*}+x_{2}^{*}, \quad x_{1}^{*} \in X^{*}\left(T^{*}, \bar{G}\right), x_{2}^{*} \in \Xi^{*}\left(T^{*}, \bar{H}\right)
$$

with $\left\|x_{1}^{*}\right\|+\left\|x_{2}^{*}\right\| \leq M\left\|x^{*}\right\|$, where the number $M$ is independent of $x^{*}$. Let $x^{*} \in D_{T^{*}}$. Then $x_{2}^{*} \in \Xi^{*}\left(T^{*}, \bar{H}\right) \subset D_{T^{*}}$ implies that $x_{1}^{*} \in D_{T^{*}}$ and hence, for every $x \in Y$, we have successively

$$
\begin{aligned}
& \left|\left\langle T^{*} x^{*}, J x\right\rangle\right|=\left|\left\langle x, T^{*} x^{*}\right\rangle\right|=\left|\left\langle x, T^{*}\left(x_{1}^{*}+x_{2}^{*}\right)\right\rangle\right|=\left|\left\langle x, T^{*} x_{2}^{*}\right\rangle\right| \\
& \leq\left\|T^{*}\left|\Xi^{*}\left(T^{*}, \bar{H}\right)\|\cdot\| x\|\cdot\| x_{2}^{*}\|\leq M\| T^{*}\right| \Xi^{*}\left(T^{*}, \bar{H}\right)\right\| \cdot\|x\| \cdot\left\|x^{*}\right\| .
\end{aligned}
$$

Thus, $\left\langle T^{*} x^{*}, J x\right\rangle$ is a bounded linear functional of $x^{*}$ and hence $J x \in$ $D_{T^{* *}}$. By Theorem 2.7, $J x \in J D_{T}$, i.e. $x \in D_{T}$. Consequently, $Y \subset D_{T}$. Since $Y$ satisfies all hypotheses of Lemma 2.2, $T / Y$ is closable and

$$
(T / Y)^{*} \cong T^{*}\left|Y^{\perp}=T^{*}\right| X^{*}\left(T^{*}, \bar{G}\right) .
$$

Thus, it follows that

$$
\sigma(\overline{T / Y})=\sigma\left[(T / Y)^{*}\right]=\sigma\left[T^{*} \mid X^{*}\left(T^{*}, \bar{G}\right)\right] \subset \bar{G} \cap \sigma(T) .
$$

It remains to show that $\sigma(T \mid Y) \subset G^{c}$. By applying Theorem 1.17 to $T^{*}$, we infer that $T^{*} / X^{*}\left(T^{*}, \bar{G}\right)$ is bounded and

$$
\sigma\left[T^{*} / X^{*}\left(T^{*}, \bar{G}\right)\right] \subset G^{c} .
$$

Now, it follows from the unitary equivalence

$$
(T \mid Y)^{*} \cong T^{*} / X^{*}\left(T^{*}, \bar{G}\right),
$$

that

$$
\sigma(T \mid Y)=\sigma\left[(T \mid Y)^{*}\right]=\sigma\left[T^{*} / X^{*}\left(T^{*}, \bar{G}\right)\right] \subset G^{c}
$$

\section{The duality theorem.}

3.1. THEOREM. Given $T$, assume that (***) holds. If $T^{*}$ has the SDP then $T$ has the SDP.

Proof. Given $\left\{G_{0}, G_{1}\right\} \in \operatorname{cov} \sigma(T)$ with $G_{0} \in V_{\infty}$ and $G_{1}$ relatively compact, let $F_{0}, F_{1} \subset \mathbf{C}$ be closed such that $F_{0} \in V_{\infty}, F_{0} \subset G_{0}, F_{1} \subset G_{1}$ 
and $\left\{\right.$ Int $F_{0}$, Int $\left.F_{1}\right\} \in \operatorname{cov} \sigma(T)$. Then $H_{0}=F_{1}^{c} \in V_{\infty}, H_{1}=F_{0}^{c}$ is compact. Put

$$
Y={ }^{\perp}\left[X^{*}\left(T^{*}, \bar{H}_{0}\right)\right], \quad Z={ }^{\perp}\left[\Xi^{*}\left(T^{*}, \bar{H}_{1}\right)\right],
$$

use Theorem 2.9 and obtain

$$
Y \subset D_{T}, \quad Y \in \operatorname{Inv} T, \quad \sigma(T \mid Y) \subset H_{0}^{c}=F_{1} \subset G_{1} .
$$

Our next objective is to obtain the decomposition

$$
X=Y+Z \text {. }
$$

Since, by Theorem $2.8, X^{*}\left(T^{*}, \bar{H}_{0}\right)$ and $\Xi^{*}\left(T^{*}, \bar{H}_{1}\right)$ are closed for $\tau\left(X^{*}, X\right)$, we have

$$
Y^{\perp}=X^{*}\left(T^{*}, \bar{H}_{0}\right), \quad Z^{\perp}=\Xi^{*}\left(T^{*}, \bar{H}_{1}\right) .
$$

It follows from

$$
\begin{aligned}
& X^{*}\left(T^{*}, \bar{H}_{0}\right)=X^{*}\left[T^{*}, \bar{H}_{0} \cap \sigma(T)\right], \\
& \Xi^{*}\left(T^{*}, \bar{H}_{1}\right)=\Xi^{*}\left[T^{*}, \bar{H}_{1} \cap \sigma(T)\right],
\end{aligned}
$$

$\left[\bar{H}_{0} \cap \sigma(T)\right] \cap\left[\bar{H}_{1} \cap \sigma(T)\right]=\varnothing$, and Theorem 1.12, that $X^{*}\left(T^{*}, \bar{H}_{0}\right)+$ $\Xi^{*}\left(T^{*}, \bar{H}_{1}\right)$ is a direct sum and

$$
X^{*}\left(T^{*}, \bar{H}_{0} \cup \bar{H}_{1}\right)=X^{*}\left(T^{*}, \bar{H}_{0}\right) \oplus \Xi^{*}\left(T^{*}, \bar{H}_{1}\right) .
$$

Apply [4, IV. Theorem 4.8] to $Y$ and $Z$, as defined by (3.1), and infer that $Y+Z$ is closed. On the other hand,

$$
(Y+Z)^{\perp}=Y^{\perp} \cap Z^{\perp}=X^{*}\left(T^{*}, \bar{H}_{0}\right) \cap \Xi^{*}\left(T^{*}, \bar{H}_{1}\right)=\{0\}
$$

implies (3.3).

It remains to show that

(a) $Z \in \operatorname{Inv} T$,

(b) $\sigma(T \mid Z) \subset G_{0}$.

For $x \in Z \cap D_{T}, x^{*} \in \Xi^{*}\left(T^{*}, \bar{H}_{1}\right)=Z^{\perp}$, we clearly have

$$
\left\langle T x, x^{*}\right\rangle=\left\langle x, T^{*} x^{*}\right\rangle=0
$$

and hence $T x \in Z$. This implies (3.6, a). By (3.3) and Proposition 1.14, $T \mid Z$ is densely defined and hence $(T \mid Z)^{*}$ exists. Next, we shall obtain

$$
(T \mid Z)^{*}=T^{*} / \Xi^{*}\left(T^{*}, \widetilde{H}_{1}\right)=\left(T^{*}\right)^{\hat{1}} \text {. }
$$

For $x \in Z \cap D_{T}, x^{*} \in D_{T^{*}}$ and

$$
\left(x^{*}\right)^{\wedge}=x^{*}+\Xi^{*}\left(T^{*}, \bar{H}_{1}\right) \in X^{*} / \Xi^{*}\left(T^{*}, \bar{H}_{1}\right),
$$


we have

$$
\left\langle(T \mid Z) x,\left(x^{*}\right)^{\wedge}\right\rangle=\left\langle(T \mid Z) x, x^{*}\right\rangle=\left\langle x, T^{*} x^{*}\right\rangle=\left\langle x,\left(T^{*}\right)^{\wedge}\left(x^{*}\right)^{\wedge}\right\rangle .
$$

Consequently,

$$
(T \mid Z)^{*} \supset\left(T^{*}\right)^{\wedge}
$$

To obtain the opposite of (3.8), let $x \in D_{T}$ and $\left(x^{*}\right)^{\hat{f}} \in D_{(T Z)^{*}}$. In view of (3.3), there is a number $M>0$ and a representation

$$
x=x_{1}+x_{2}, \quad x_{1} \in Y, x_{2} \in Z \quad \text { and } \quad\left\|x_{1}\right\|+\left\|x_{2}\right\| \leq M\|x\| .
$$

Then, for every $x^{*} \in\left(x^{*}\right)^{\hat{n}}\left(\in D_{(T Z)^{*}}\right)$, we have successively:

$$
\begin{aligned}
\left|\left\langle T x, x^{*}\right\rangle\right| & \leq\left|\left\langle T x_{1}, x^{*}\right\rangle\right|+\left|\left\langle T x_{2}, x^{*}\right\rangle\right| \\
& =\left|\left\langle(T \mid Y) x_{1}, x^{*}\right\rangle\right|+\left|\left\langle(T \mid Z) x_{2},\left(x^{*}\right)^{\wedge}\right\rangle\right| \\
& \leq\|T \mid Y\| \cdot\left\|x_{1}\right\| \cdot\left\|x^{*}\right\|+\left\|x_{2}\right\| \cdot\left\|(T \mid Z)^{*}\left(x^{*}\right)^{\wedge}\right\| \\
& \leq M\left\{\|T \mid Y\| \cdot\left\|x^{*}\right\|+\left\|(T \mid Z)^{*}\left(x^{*}\right)^{\wedge}\right\|\right\}\|x\| .
\end{aligned}
$$

Thus, $\left\langle T x, x^{*}\right\rangle$ is a bounded linear functional of $x$. Consequently, $x^{*} \in$ $D_{T^{*}}$ and hence $\left(x^{*}\right)^{\hat{n}} \in D_{\left(T^{*}\right)}$. In view of (3.8), (3.7) is obtained. Now Theorem 1.17 applied to $T^{*}$, gives

$$
\sigma\left[\left(T^{*}\right)^{\wedge}\right] \subset H_{1}^{c}
$$

and hence $(3.6, b)$ follows by

$$
\sigma(T \mid Z)=\sigma\left[(T \mid Z)^{*}\right]=\sigma\left[\left(T^{*}\right)^{\wedge}\right] \subset H_{1}^{c}=F_{0} \subset G_{0} .
$$

By (3.3), (3.2) and (3.6), $T$ has the SDP.

The combination of Theorems 2.1 and 3.1 gives

3.2. COROllary. Given $T$, assume that condition $(* * *)$ holds. Then $T$ has the SDP iff $T^{*}$ has the SDP.

\section{REFERENCES}

[1] C. Apostol, Roots of decomposable operator-valued analytic functions. Rev. Roumaine Math. Pures Appl., 13 (1968), 433-438.

[2] I. Bacalu, Some properties of decomposable operators, Rev. Roumaine Math. Pures Appl., 21 (1976), 177-194.

[3] C. Foiaş, Spectral maximal spaces and decomposable operators in Banach spaces. Acta Math. (Basel), 14 (1963), 147-158.

[4] T. Kato, Perturbation Theory for Linear Operators. Springer-Verlag, New York, 1966. 
[5] B. Nagy, Operators with the spectral decomposition property are decomposable. Studia Sci. Math. Hungar., 13 (1978), 429-432.

[6] A A spectral residuum for each closed operator. Topics in Modern Operator Theory: Advances and Applications. Vol. 2. Birkhäuser Verlag 1981, 221-238.

[7] M. Radjabalipour, Equivalence of decomposable and 2-decomposable operators. Pacific J. Math., 77 (1978), 243-247.

[8] F.-H. Vasilescu, Residually decomposable operators in Banach spaces. Tôhoku Math. J., 21 (1969), 509-522.

[9] On the residual decomposability in dual spaces. Rev. Roumaine Math. Pures Appl., 16 (1971), 1573-1587.

[10] Wang Shengwang and Liu Guangyu, On the duality theorems of S-decomposable operators, J. Math. Anal. Appl., in print.

[11] Zhang Dianzhou and Wang Shushi, On closed decomposable operators. Chinese Ann. Math., in print.

Received September 24, 1982 and in revised form September 14, 1983.

TEMPLE UNIVERSITY

PhiladelPhia, PA 19122

AND

NANJING UNIVERSITY

NANJING, CHINA 



\section{PACIFIC JOURNAL OF MATHEMATICS \\ EDITORS}

DONALD BABBITt (Managing Editor)

University of California

Los Angeles, CA 90024

J. Dugundu

University of Southern California

Los Angeles, CA 90089-1113

R. FINN

Stanford University

Stanford, CA 94305

HERMANN FLASCHKA

University of Arizona

Tucson, AZ 85721
C. C. MOORE

University of California

Berkeley, CA 94720

ARTHur Ogus

University of California

Berkeley, CA 94720

Hugo RossI

University of Utah

Salt Lake City, UT 84112

H. SAMELSON

Stanford University

Stanford, CA 94305

ASSOCIATE EDITORS

R. ARENS

E. F. BECKENBACH

B. H. NeumanN (1906-1982)

F. WOLF

K. YoshidA

\section{SUPPORTING INSTITUTIONS}

UNIVERSITY OF ARIZONA

UNIVERSITY OF BRITISH COLUMBIA

UNIVERSITY OF OREGON

CALIFORNIA INSTITUTE OF TECHNOLOGY

UNIVERSITY OF CALIFORNIA

MONTANA STATE UNIVERSITY

UNIVERSITY OF SOUTHERN CALIFORNIA

UNIVERSITY OF NEVADA, RENO

STANFORD UNIVERSITY

UNIVERSITY OF HAWAII

NEW MEXICO STATE UNIVERSITY

UNIVERSITY OF TOKYO

UNIVERSITY OF UTAH

OREGON STATE UNIVERSITY

WASHINGTON STATE UNIVERSITY

UNIVERSITY OF WASHINGTON 


\section{Pacific Journal of Mathematics}

Vol. 114, No. $1 \quad$ May, 1984

David Marion Arnold and Charles Irvin Vinsonhaler, Typesets and cotypesets of rank-2 torsion free abelian groups $\ldots \ldots \ldots \ldots \ldots \ldots \ldots 1$

Duncan Alan Buell and Richard Howard Hudson, Solutions of certain

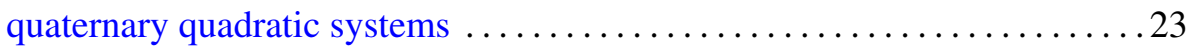

Hans Delfs and Manfred Knebusch, Separation, retractions and homotopy

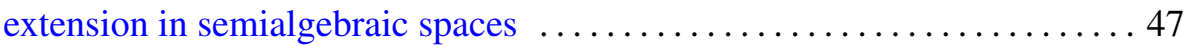

I. Erdélyi and Sheng-Wang Wang, A spectral duality theorem for closed

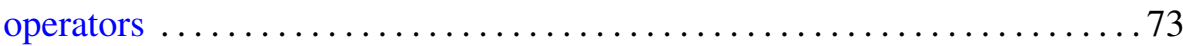

Theodore William Gamelin, Weak compactness of representing measures for $R(K)$

Kenneth R. Goodearl and T. H. Lenagan, Krull dimension of

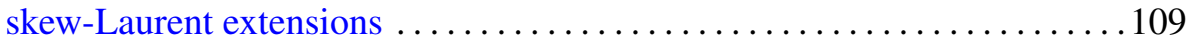

Daniel Joseph Gross, Compact quotients by $\mathbf{C}^{*}$-actions . . . . . . . . . . . 149

Goo Ishikawa, Satoshi Koike and Masahiro Shiota, Critical value sets of

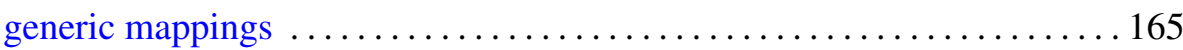

Hong Oh Kim, Derivatives of Blaschke products $\ldots \ldots \ldots \ldots \ldots \ldots \ldots \ldots \ldots$

Erhard Luft and Denis Karmen Sjerve, 3-manifolds with subgroups

$Z \oplus Z \oplus Z$ in their fundamental groups $\ldots \ldots \ldots \ldots \ldots \ldots \ldots \ldots \ldots \ldots \ldots \ldots$

George Clifford Nelson, Boolean powers, recursive models, and the Horn

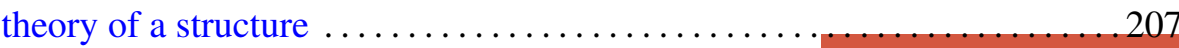

W. J. Phillips, Flow under a function and discrete decomposition of properly infinite $W^{*}$-algebras

Teodor C. Przymusiński, A solution to a problem of E. Michael ... 235

Bruce Harvey Wagner, Derivations of quasitriangular algebras 243 